\title{
MEDIUM ACCESS CONTROL WITH SCHEDULED GROUP CONTENTION FOR MASSIVE M2M DEVICES
}

\author{
Joo Rak Kang ${ }^{1}$ and Tae-Jin Lee ${ }^{2}$ \\ ${ }^{1}$ Samsung Electro-Mechanics Co., Ltd., Suwon, Korea \\ College of Information and Communication Engineering, \\ Sungkyunkwan University, Suwon, Korea \\ jrkang@skku.edu \\ ${ }^{2}$ College of Information and Communication Engineering, \\ Sungkyunkwan University, Suwon, Korea \\ tjlee@skku.edu
}

\begin{abstract}
In a dense Machine-to-Machine (M2M) network, a large number of stations contend to achieve transmission opportunity and it creates a critical congestion problem. To solve this issue, a group-based contention Medium Access Control (MAC) protocol is introduced. Stations are divided into small numbers of groups and only one station in each group will contend as a group leader to achieve the reserved time interval dedicated to a winner group. It can reduce the contention overhead and lessen the congestion problem. In this paper, we propose Scheduled Group Contention MAC (SGCMAC) protocol to enhance the group-based contention MAC. The proposed SGCMAC protocol divides groups based on the traffic categories of stations and schedules the contention groups to reduce the contention overhead. We also propose an efficient resource management mechanism in the group grant time to prevent the waste of time caused by idle stations. Simulations with IEEE 802.11ah parameters demonstrate that our proposed SGCMAC has performance gains over other group-based contention MAC protocols.
\end{abstract}

\section{KEYWORDS}

Medium Access Control (MAC), Machine-to-Machine, Grouping, Wireless LAN, Random Access Protocols

\section{INTRODUCTION}

Internet of Things (IoT) is a new paradigm for the future of the Internet. IoT will integrate a variety of sensors, actuators, smart devices and things through the Internet, and distributed smart services will be created to change our daily life. Machine-to-Machine (M2M) communications is considered as a basic communication technology for the realization of IoT. M2M communications involves information exchange among machines without any human interaction. M2M networks are expected to be widely utilized in many fields of various IoT applications such

David C. Wyld et al. (Eds) : NETCOM, NCS, WiMoNe, CSEIT, SPM - 2015

pp. 39-49, 2015. () CS \& IT-CSCP 2015

DOI : $10.5121 /$ csit.2015.51604 
as public services, industrial automations, health care, intelligent transport systems, smart grids, and agricultural networks [1]-[5].

M2M networks have unique characteristics which are quite different from conventional networks. In M2M networks, tremendous number of devices may be involved in the service coverage and concurrent network access attempts may occur from the devices. Typically, the amount of data generated from each device may be small and data may be generated infrequently. If, however, many devices participate in and generate data at the same time, it may cause a critical congestion problem. Also, in M2M networks, high level of system automation, in which devices and systems exchange and share data automatically, is required. Therefore, congestion control is a fundamental issue in M2M networks. Especially, from the Medium Access Control (MAC) layer perspective, efficient access control and management of network resources is one of the most challenging issues in M2M networks [2]. In addition to congestion control and scalability, an M2M network has to support various traffic categories and quality of service (QoS) requirements. In this context, it is important how to distribute traffic and network access attempts subject to QoS requirements, which is the motivation to our work.

To address the issue, group-based access control schemes have been introduced in the literature [6]-[9]. The authors in [6] introduce a grouping-based radio resource management for 3GPP M2M communications. In [7], IEEE 802.11ah task group proposes Restricted Access Window (RAW) to allow contending channel only for a small number of stations. The group-based contention MAC protocols, a hybrid scheme to combine the advantages of contention-based and contention-free protocols, are presented, Group-based Medium Access Control (GMAC) [8] and Group Leader DCF-TDMA (DCFT) [9]. In GMAC and DCFT, all stations are divided into small numbers of groups and only one station in each group, called a group leader, involves in channel contention. If a group leader wins the competition, a certain time duration is reserved for its associated group and resources are allocated to the group members according to a contention-free protocol.

In this paper, we propose an enhanced group-based contention MAC protocol, Scheduled Group Contention MAC (SGCMAC) protocol, which is more suitable for M2M networks with heterogeneous M2M traffics. Our SGCMAC divides the machine type communications (MTC) stations into small numbers of groups according to their traffic categories, e.g., applications, trigger event types and periods of traffic. The proposed group-based resource allocation scheme is very efficient since the temporal correlation among the group members of a group is typically very high. The Access Point (AP) can schedule the contention time duration of each group based on its traffic characteristics. It can spread the contention groups over a wide range of time interval. Therefore, our proposed SGCMAC is not only efficient in reducing the contention overhead in massive M2M networks but also in being applicable to M2M networks with heterogeneous traffics. We develop an efficient mechanism to detect active stations and idle stations in the reserved time duration for a winner group, which removes the waste time caused by idle stations during the reserved time. The rest of this paper is organized as follows. In Section

II and Section III, we describe the system model and the proposed SGCMAC protocol. Section IV presents simulation results to evaluate performance of the proposed protocol. Finally, Section $\mathrm{V}$ concludes the paper.

\section{SYSTEM MODEL}

We consider a single-hop wireless network consisting of one AP and $n$ MTC stations. The network works based on the IEEE 802.11ah MAC and Physical (PHY) layer. Each device 
follows the Distributed Coordinate Function (DCF) to access the medium and operates in the sub$1 \mathrm{GHz}$ frequency band as described in [7], [10]. The MTC stations in a network have two types of operation status, i.e., active status and idle status. When a device is in the active status, it has upload data. We assume a single data buffer in an M2M device. If a active station successfully transmits data, this station goes to idle status. The events are assumed to arrive at each station by a Poisson process with the average arrival rate $\lambda$. Fig. 1 shows an example of DCF operation that active stations content the channel access according to DCF rules.

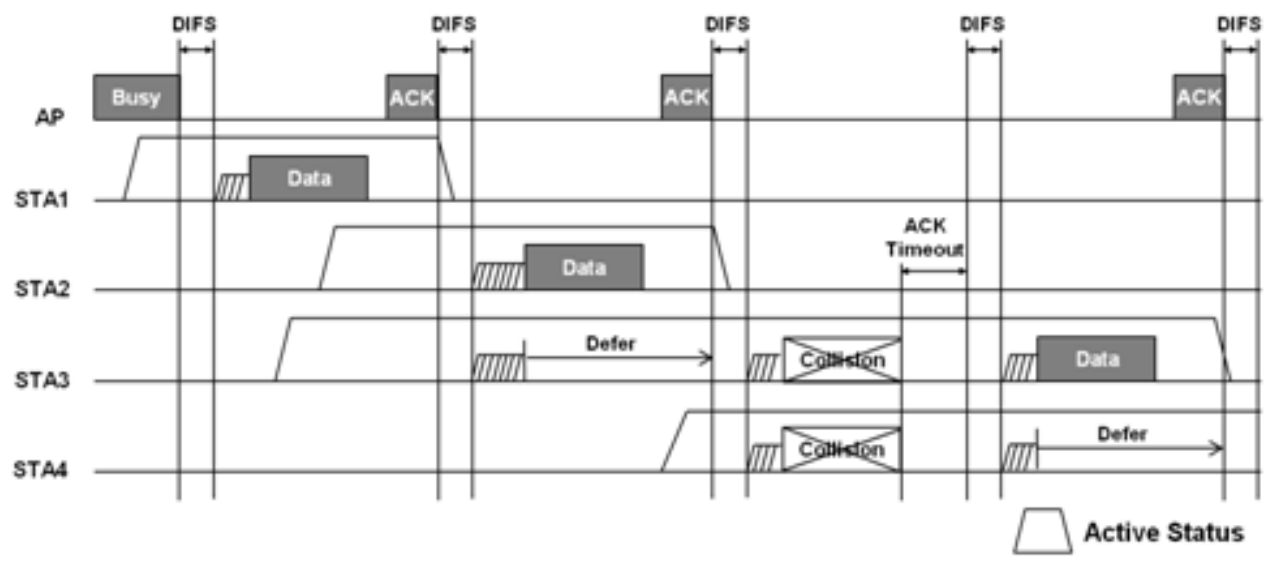

Fig. 1 An example of IEEE 802.11 Distributed Coordinate Function (DCF) operation.

\section{PROPOSED SGCMAC PROTOCOL}

In this section we describe our proposed SGCMAC protocol. The related grouping rule and the group leader selection algorithm are also introduced. To address the contention problem in a dense M2M network, we divide all MTC devices into several groups according to their traffic categories. The AP assigns the group leader role to a member in each group and schedules time intervals for some of the group leaders to coordinate the channel access. We call this specific time interval Group Access Window (GAW). The scheduled group leaders contend in a GAW by a modified DCF scheme. When a group leader wins the contention, the AP assigns time slots to the group members to allow data transmission by the group period granting procedure. The procedure for the SGCMAC protocol is shown in Fig. 2.

\subsection{Group Access Window}

The AP allocates a particular time period called GAW in a beacon interval for the group-based channel access. The information of a GAW such as the start time and the duration of the GAW and the scheduled groups for the GAW is announced through the Scheduled Group Indication Map (SGIM) Information Element in a beacon. The group leaders in the SGIM start contention to acquire a Group Granted Period (GGP) at the GAW start time.

The contention procedure among the group leaders is similar to the Request to Send (RTS)/Clear to Send (CTS) procedure based on the DCF scheme. After a random backoff, a group leader sends a Group Access Request (GAR) frame to the AP. If there is no collision, the AP responds by the Group Access Grant (GAG) frame. Since all the active devices in a network as well as the contending group leaders can hear the GAG frame, the winner group is allowed the GGP, defined in the duration field of the GAG frame. The AP checks the active group members in the winner group and assigns time slots to the active stations by Time Division Multiple Access (TDMA) 
scheme. If the total duration of the assigned time slots is less than GGP in the duration field of the GAG frame, the AP broadcasts the Group Access End (GAE) frame after the data exchange of the last active group member to terminate GGP. After the GGP, the group leaders resume contention again to access the channel until the end of GAW.

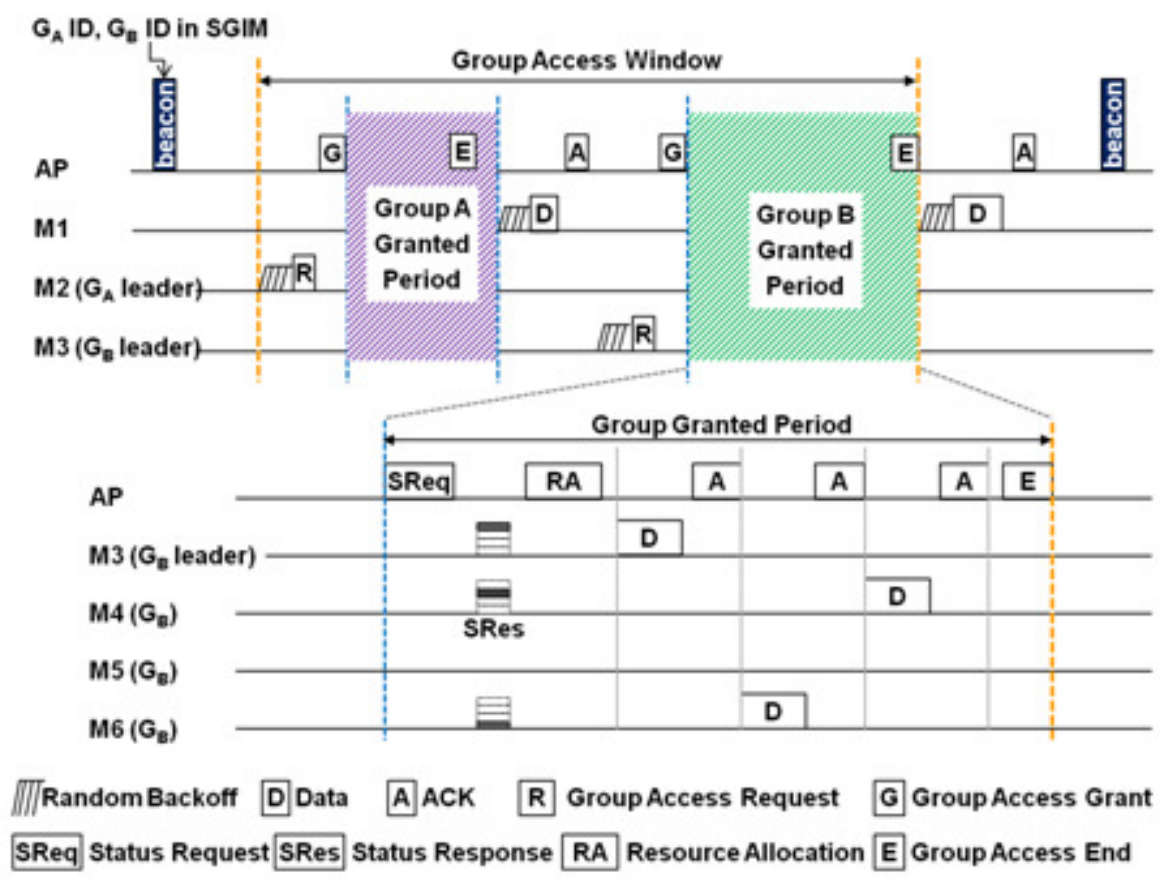

Fig. 2. Group Access Window (GAW) and Scheduled Group Contention MAC (SGCMAC) protocol.

The GAW has advantages. Because an AP can schedule a contention group at a particular GAW, M2M traffic and contention will be spread across beacon periods and QoS requirements of each group are able to be supported. Moreover, M2M devices can coexist with legacy devices since the mechanism of channel reservation for a group allows the protection mechanism.

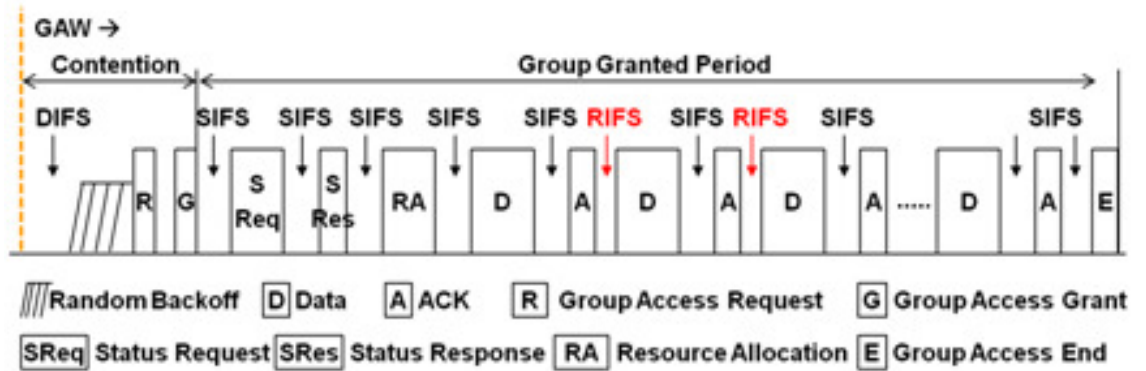

(a) Transmission of stations in a GGP 


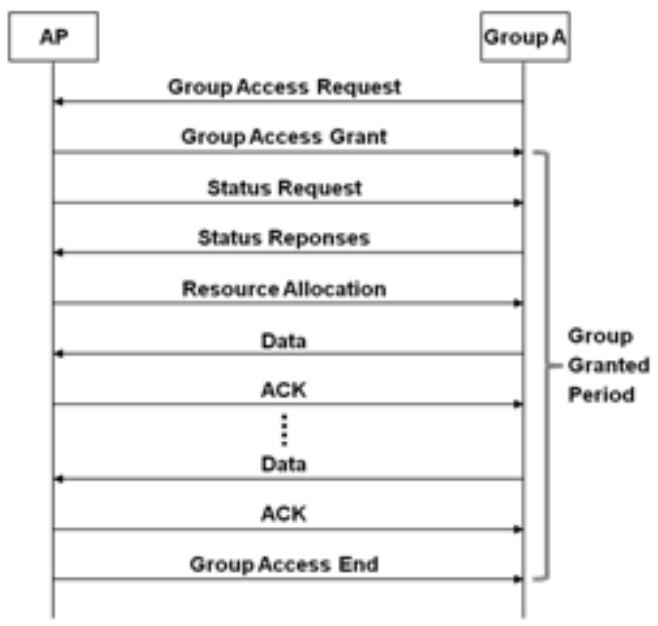

(b) Frame exchange sequence during GGP

Fig. 3. An example of Group Granted Period (GGP).

\subsection{Transmission of Stations in a GGP}

When a group leader wins the contention, the winner group gets the dedicated channel access time called GGP. The transmission time for each station in a winner group is assigned by an AP using a TDMA scheme. However, if a station is not activated in GGP, the time slot for this station will be wasted. In order to prevent this waste, the AP has to check whether each station is in the active status or not before assigning time slots. This status check procedure may be an overhead. To minimize this overhead, we use a signal multiplexing scheme in the frequency domain as in [13]. When the GGP starts, the AP transmits the Status Request (SReq) frame to the group members. The SReq frame includes the sub-carrier assignment map for each group member. Active group members can hear the SReq frame and knows the sub-carrier assigned to itself. After Short Inter-Frame Space (SIFS), the active group members respond by sending Status Response (SRes) by using single tone signals assigned by the AP. Based on this SRes, the AP will be able to assign time slots to the active group members without wasting time. Since we assume that the group members have fixed data size, the AP can schedule the time slots for each of the active members by announcing the order of the active members. Then the AP transmits a Resource Allocation (RA) frame including the schedule information.

In our protocol, a time slot is assigned for a particular station. So, very small time interval larger than the propagation delay is enough to separate data exchange sequences of each active member. In this paper, this inter-time slot of a member is designed and utilized as in Reduced Inter-Frame Space (RIFS) defined in the IEEE 802.11 standard. Fig. 3 shows an example of GGP. The total channel occupation time for one group access consists of the time duration to check the active stations and the scheduled time slots for data transmission. Let $T_{C}$ and $T_{S}$ denote the time durations to confirm the active stations and the scheduled time slots, respectively.

$$
T_{C}=T_{S R E Q}+T_{S R E S}+T_{R A}+S I F S \times 4
$$

where $T_{S R E Q}, T_{S R E S}$, and $T_{R A}$ denote the duration for the SReq frame, the SRes, and the RA frame, respectively. 


$$
\begin{gathered}
T_{S}=\left(T_{D}+S I F S+T_{A}+R I F S\right) \times N_{A G M} \\
+\left(S I F S-R I F S+T_{E}\right)
\end{gathered}
$$

where $T_{D}, T_{A}$ and $T_{E}$ denote the duration for data, acknowledgment (ACK), and GAE frame, respectively. $N_{A G M}$ denotes the number of active stations in a specific GGP. The total channel occupation time $T_{\text {Grant }}$ for one group access is then calculated as follows.

$$
T_{\text {Grant }}=T_{C}+T_{S}
$$

\subsection{Grouping and Group Leader Selection Algorithm}

We divide MTC stations into groups according to the traffic patterns of stations while GMAC and DCFT make groups based on the region or the coverage of nodes. After grouping, there is only a small number of stations involved in contention at a certain time duration and the hidden terminal issue may be reduced by the RTS/CTS mechanism as defined in [10]. If the temporal correlation between group member stations is low, it will adversely affect the channel efficiency of the group-based contention MAC.

A traffic pattern of each MTC station depends on its application or service type. The traffic of an M2M application or service is classified into Fixed-Scheduling (FS) or Event-Driven (ED) [11]. Furthermore, it can be categorized into periodic, trigger event, or random based on its application [6], [12]. When an MTC station associates with the AP, a station informs its traffic category and application type to the AP. The AP assigns this station to a proper group using the Group ID (GID) and the Group Member ID (GMID). In the proposed protocol, we limit the maximum number of group members to a number less than the number of sub-carriers for efficient group management. Accordingly, it can create multiple groups with the same traffic category.

One of the group members is selected as a group leader by the AP. The group leader consumes more power than the other group members due to the contention for its GGP. To prevent too early burning out of a group leader station, the AP may change the group leader every GGP. The AP chooses the next group leader among the active group members in a round-robin manner and sends its information in the RA frame.

\section{SIMULATION RESULTS}

In this section, we present the simulation results of the proposed SGCMAC protocol, GMAC [8] and DCFT [9]. We develop our own simulator which have been performed with the PHY and MAC layer characteristics based on the IEEE 802.11ah standard [7] as in Table 1. We assume that the number of stations in each group is fixed to 50. We measure the temporary reservation time for a winner group succeeded in the group contention and the length of group management frames such as the Polling frame in GMAC and the SReq frame and the RA frame in SGCMAC. The data rate of stations in the simulations is $650 \mathrm{Kbps}$ defined as the basic data rate in IEEE 802.11ah. 
Table 1. Simulation Parameters

\begin{tabular}{|c|c|l|}
\hline Parameter & Value & \multicolumn{1}{|c|}{ Description } \\
\hline aSlotTime & $52 \mu \mathrm{s}$ & Backoff slot time \\
\hline aRIFSTime & $20 \mu \mathrm{s}$ & Reduced Inter-Frame Space \\
\hline aSIFSTime & $160 \mu \mathrm{s}$ & Short Inter-Frame Space \\
\hline aDIFSTime & $264 \mu \mathrm{s}$ & DCF Inter-Frame Space \\
\hline$C W_{\min }$ & 16 & Minimum contention window size \\
\hline$C W_{\max }$ & 512 & Maximum contention window size \\
\hline$N_{G M}$ & 50 & Number of group members \\
\hline Data Rate & $650 \mathrm{Kbps}$ & S1G MCS0 for 2MHz channel \\
\hline Payload & $512 \mathrm{Bytes}$ & Data payload size \\
\hline MAC Overhead & $22 \mathrm{Bytes}$ & S1G short MAC header+FCS \\
\hline$T_{P H Y}$ & $280 \mu \mathrm{s}$ & PHY header \\
\hline$T_{R T S}$ & $560 \mu \mathrm{s}$ & RTS, DCFT poll, GAR frames \\
\hline$T_{C T S}$ & $480 \mu \mathrm{s}$ & CTS, CFEND, GAG, GAE frames \\
\hline$T_{R A}$ & $1120 \mu \mathrm{s}$ & SReq, RA, GMAC polling frames \\
\hline$T_{S R E S}$ & $40 \mu \mathrm{s}$ & SRes frame \\
\hline$T_{A C K}$ & $240 \mu \mathrm{s}$ & ACK frame \\
\hline & &
\end{tabular}

\subsection{Performance in Homogeneous Traffic}

Fig. 4 and Fig. 5 show the performance under the homogeneous traffic condition. The number of stations associated with the AP is 4000 and they are divided into 80 groups. All stations belong to one traffic category with the same event arrival rate. Our proposed SGCMAC shows gains in throughput and delay compared with the other group-based contention MAC schemes.

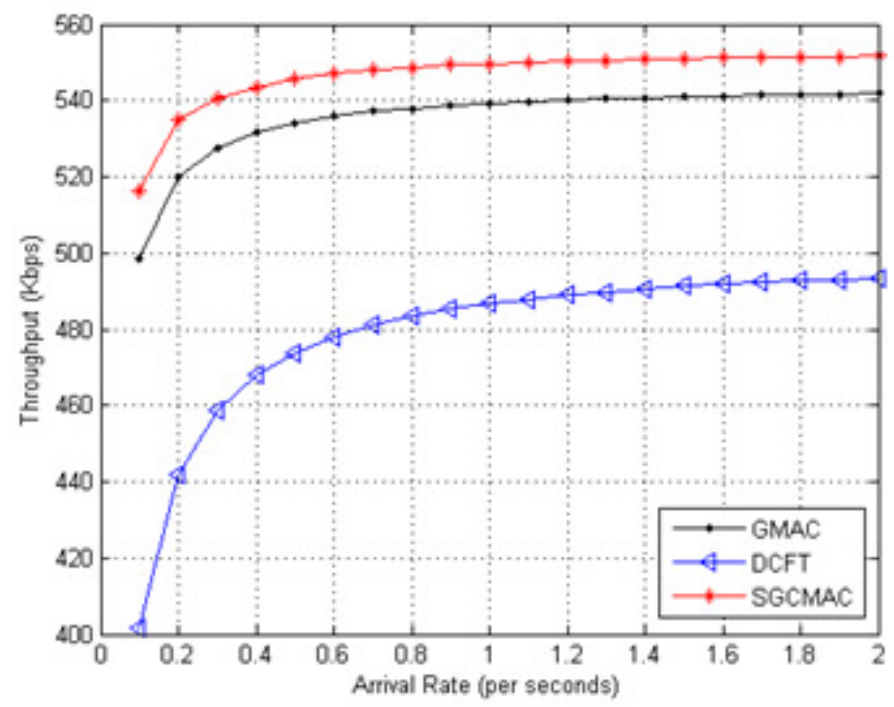

Fig. 4. Throughput for varying arrival rates under the homogeneous traffic condition. 


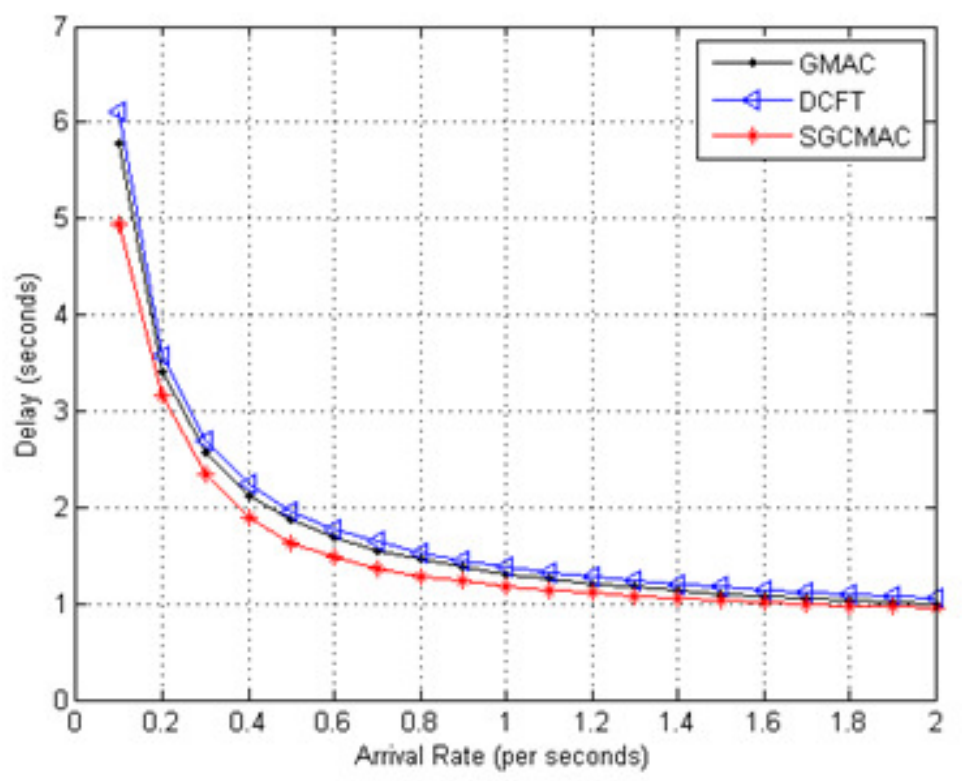

Fig. 5. Average delay for varying arrival rates under the homogeneous traffic condition.

Fig. 4 shows the throughput for varying event arrival rates from 0.1 to 2.0. As the arrival rate increases above 1.0, the network traffic reaches the saturation condition. Then almost all stations are in the active status whenever their groups achieve the reserved time slots for the group members. In this case, the contention overhead and the static overhead of each MAC protocol mainly affects the throughput. If the arrival rate decreases, stations stay in the idle status for a long time, and a large portion of the reserved time interval for a winner group will be wasted. In order to reduce this waste, it is important to efficiently detect idle stations in the reserved time interval for a winner group. Our proposed SGCMAC exhibits better performance than the others in terms of channel efficiency and throughput.

The average delay of stations is presented in Fig. 5. Our proposed SGCMAC has a gain of 850ms compared with the others at the arrival rate of 0.1. The proposed SGCMAC protocol has lower contention overhead and less waste of time in the granted group duration than the others. When the network traffic reaches its saturation condition, the average contention overhead and the static MAC and PHY overhead only affects the delay. In this case, our proposed SGCMAC also shows lower delay than the others.

\subsection{Performance in Heterogeneous Traffic}

In Fig. 6 and Fig. 7, the throughput and the delay are presented for varying numbers of stations under the heterogeneous traffic condition. There are 20 traffic categories which are classified according to their arrival rates from 0.01 to 0.2 . All stations are grouped into 20 traffic categories such that equal number of stations is assigned to each of the categories. 


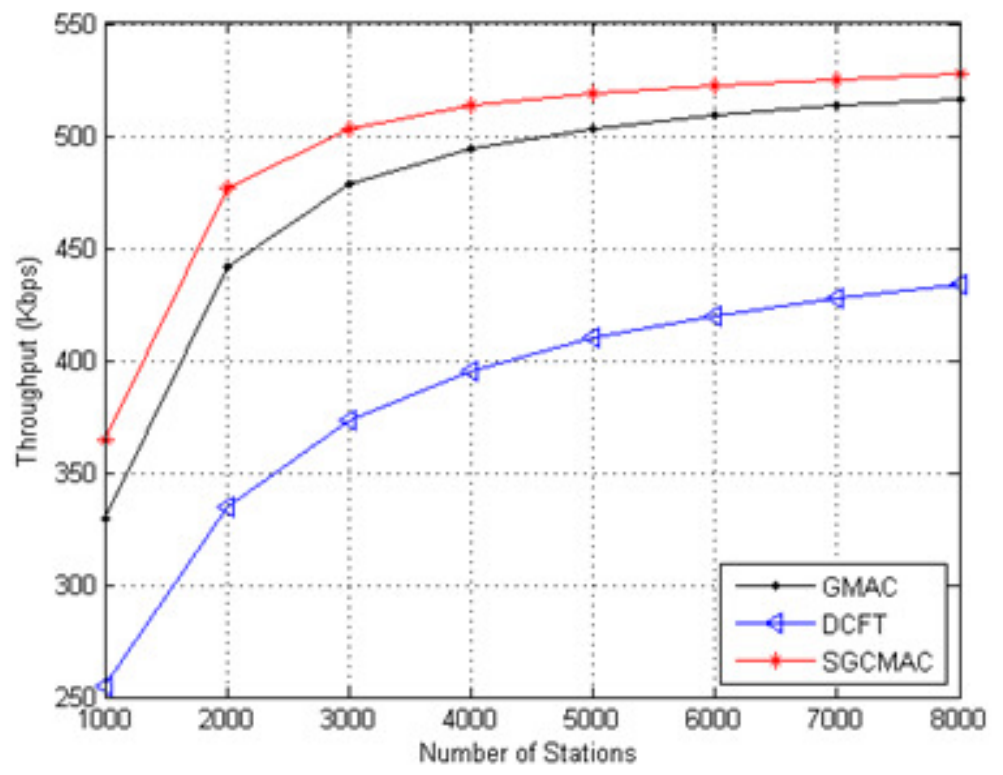

Fig. 6. Throughput for varying numbers of stations under the heterogeneous traffic condition.

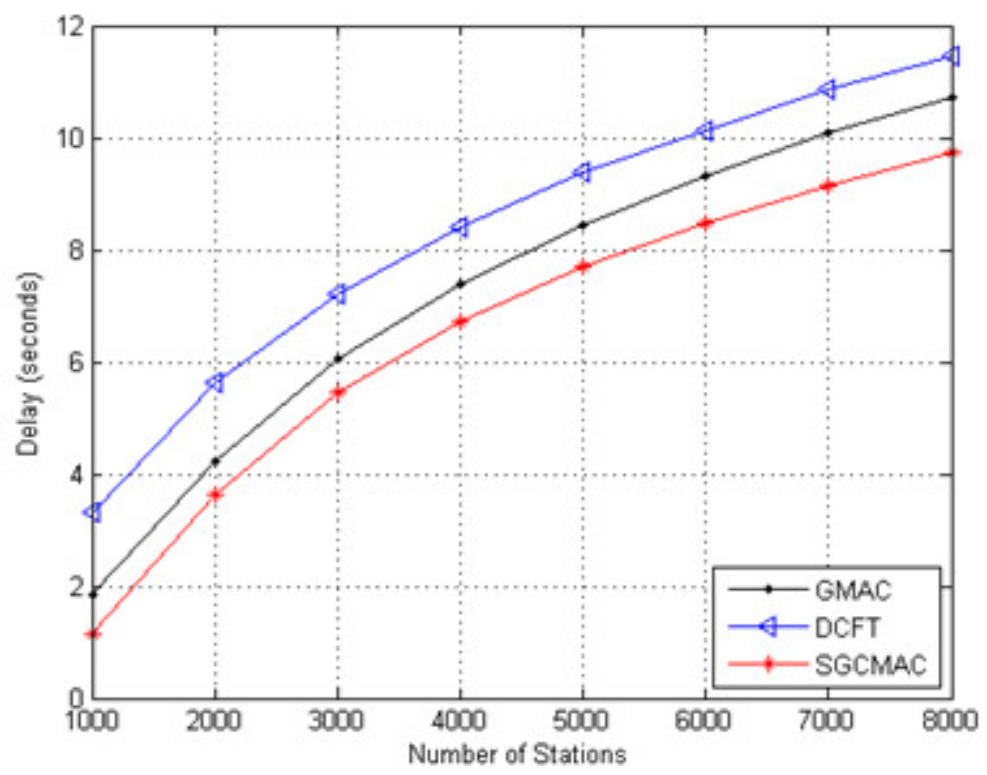

Fig. 7. Average delay for varying number of stations under heterogeneous traffic condition.

The throughput for varying number of stations is shown in Fig. 6. The throughput of our proposed SGCMAC is higher than those of GMAC and DCFT. The average gain of the throughput is $20 \mathrm{Kbps}$ and $113 \mathrm{Kbps}$ compared with those of GMAC and DCFT, respectively. If the number of groups increases due to varying number of stations, the contention overhead in DCF also increases, which then decreases throughput. In the simulation, the arrival rate of each group is lower than 0.2. So most stations stay in the idle status for a long time. However, if the channel access interval of each group gets longer due to increasing number of contention groups, the member stations in the active status will increase in the reserved time duration for a winner 
group. Then the network throughput increases as the number of stations increases as shown in Fig. 6.

In this traffic condition, the delay is a more important performance metric. The average delay of stations is presented in Fig. 7. The delay of SGCMAC is better than those of the other groupbased contention MAC protocols. The delay of SGCMAC is $600 \mathrm{~ms}$ to 2.2 seconds lower than those of the others. In our proposed SGCMAC, the contention overhead does not change regardless of the number of stations since the AP is able to schedule a certain number of contention groups in GAW. In the simulation, the number of contention groups in every GAW is controlled to 10. Since the proposed SGCMAC keeps the low contention overhead and minimizes the waste time in the reserved time duration for a winner group, it shows lower delay over the whole range of the number of stations than the others.

\section{CONCLUSION}

In this paper, we have proposed a group-based contention MAC protocol for a dense M2M network. Our proposed SGCMAC divides groups based on the traffic categories of stations and schedules group contention time slots. It can reduce the contention overhead for channel access and solve a congestion problem in a dense M2M network. In addition, we propose an efficient mechanism to determine active stations in the reserved time duration for a winner group and resources are allocated to reside within the actual transmission time. We can reduce the waste time caused by idle stations in the group access time. Through simulations with the IEEE 802.11ah MAC and PHY parameters, we evaluate the throughput and delay performance of our proposed SGCMAC compared to GMAC and DCFT. The simulation results demonstrate that our proposed SGCMAC has performance gains compared with the other group-based contention MAC protocols. As a future work, we would like to evaluate our SGCMAC protocol performance based on real deployed M2M network traffic model.

\section{ACKNOWLEDGMENT}

This work was supported by the National Research Foundation of Korea (NRF) grant funded by the Korean government (MSIP) (2014R1A5A1011478).

\section{REFERENCES}

[1] L. Atzori, A. Iera, and G. Morabito, "The Internet of Things: A Survey," Computer Networks, vol. 54, no. 15, pp. 2787-2805, Oct. 2010.

[2] A. Rajandekar and B. Sikdar, "A Survey of MAC Layer Issues and Protocols for Machine-toMachine Communications," IEEE Internet of Things Journal, vol. 2, no. 2, pp. 175-186, Apr. 2015.

[3] J. Kim, J. Lee, J. Kim, and J. Yun, "M2M Service Platforms: Survey, Issues, and Enabling Technologies," IEEE Communications Surveys \& Tutorials, vol. 16, no. 1, Feb. 2014.

[4] L. Karim, A. Anpalagan, N. Nasser, and J. Almhana, "Sensor-based M2M Agriculture Monitoring Systems for Developing Countries: State and Challenges," Network Protocols and Algorithms, vol. 5, no. 3, pp. 68-86, 2013. 
[5] A. Reche, S. Sendra, J. R. Diza, and J. Lloret, "A Smart M2M Deployment to Control the Agriculture Irrigation," Ad-hoc Networks and Wireless of the series Lecture Notes in Computer Science, vol. 8629, pp. 139-151, Feb. 2015.

[6] S.-Y. Lien, K.-C. Chen, and Y. Lin, "Toward Ubiquitous Massive Accesses in 3GPP Machine-toMachine Communications,” IEEE Communication Magazine, vol. 49, no. 4, pp. 66-74, Apr. 2011.

[7] IEEE P802.11ah Draft Ver.5.0, IEEE Std. 802.11 TGah, Mar. 2015.

[8] Z. Abichar and J. M. Chang, "Group-Based Medium Access Control for IEEE 802.11n Wireless LANs,” IEEE Trans. Mobile Computing, vol. 12, no. 2, pp. 304-317, Feb. 2013.

[9] Y. Yang and S. Roy, "Grouping-Based MAC Protocols for EV Charging Data Transmission in Smart Metering Network," IEEE J. Selected Areas in Communications, vol. 32, no. 7, pp. 1328-1343, Jul. 2014.

[10] IEEE Std 802.11-2012, Part 11: Wireless LAN Medium Access Control (MAC) and Physical Layer (PHY) Specifications, IEEE, Mar. 2012.

[11] O. Al-Khatib, W. Hardjawana, and B. Vucetic, "Traffic Modeling for Machine-to-Machine (M2M) Last Mile Wireless Access Networks," in Proc. of IEEE Global Communications Conference (GLOBECOM), pp. 1199-1204, Dec. 2014.

[12] R. Liu, W. Wu, H. Zhu, and D. Yang, "M2M-Oriented QoS Categorization in Cellular Network," in Proc. of 7th International Conference on Wireless Communications, Networking and Mobile Computing (WiCOM), Sep. 2011.

[13] S. Sen, R. Roy Choudhury, and S. Nelakuditi, "No Time to Countdown: Migrating Backoff to the Frequency Domain," in Proc. of 17th Annual International Conference on Mobile Computing and Networking (MobiCom), pp. 241-252, Sep. 2011. 